\title{
The use of iron charge state changes as a tracer for solar wind entry and energization within the magnetosphere
}

\author{
T. A. Fritz ${ }^{1}$, T. H. Zurbuchen ${ }^{2}$, G. Gloeckler ${ }^{2,3}$, S. Hefti ${ }^{2}$, and J. Chen ${ }^{1}$ \\ ${ }^{1}$ Boston University, Center for Space Physics, 725 Commonwealth Ave., Boston, MA 02215, USA \\ ${ }^{2}$ University of Michigan, Dept AO \& SS, Ann Arbor, MI, 48109, USA \\ ${ }^{3}$ University of Maryland, Dept Physics, College Park, MD 20742-2425, USA
}

Received: 17 September 2002 - Revised: 15 April 2003 - Accepted: 17 April 2003

\begin{abstract}
The variation of the charge state of iron $[\mathrm{Fe}]$ ions is used to trace volume elements of plasma in the solar wind into the magnetosphere and to determine the time scales associated with the entry into and the action of the magnetospheric energization process working on these plasmas. On 2-3 May 1998 the Advanced Composition Explorer (ACE) spacecraft located at the L1 libration point observed a series of changes to the average charge state of the element $\mathrm{Fe}$ in the solar wind plasma reflecting variation in the coronal temperature of their original source. Over the period of these two days the average Fe charge state was observed to vary from +15 to +6 both at the Polar satellite in the high latitude dayside magnetosphere and at ACE. During a period of southward IMF the observations at Polar inside the magnetosphere of the same Fe charge state were simultaneous with those at ACE delayed by the measured convection speed of the solar wind to the subsolar magnetopause. Comparing the phase space density as a function of energy at both ACE and Polar has indicated that significant energization of the plasma occurred on very rapid time scales. Energization at constant phase space density by a factor of 5 to 10 was observed over a range of energy from a few $\mathrm{keV}$ to about $1 \mathrm{MeV}$. For a detector with a fixed energy threshold in the range from $10 \mathrm{keV}$ to a few hundred $\mathrm{keV}$ this observed energization will appear as a factor of $\sim 10^{3}$ increase in its counting rate. Polar observations of very energetic $\mathrm{O}^{+}$ions at the same time indicate that this energization process must be occurring in the high latitude cusp region inside the magnetosphere and that it is capable of energizing ionospheric ions at the same time.
\end{abstract}

Key words. Magnetospheric physics (magnetopause, cusp, and boundary layers; magnetospheric configuration and dynamics; solar wind-magnetosphere interactions)

Correspondence to: T. A. Fritz (fritz@bu.edu)

\section{Introduction}

The most striking and significant finding of the Polar satellite is that energetic particles are observed to be consistently and continuously present in the high latitude, high altitude dayside magnetosphere where they cannot be stably trapped. The source of these particles remains a topic of debate and scientific discussion. There are three sources under active consideration as the supplier of these particles. Source 1 has the particles produced upstream of the magnetosphere at the location of the bowshock when a "quasi-parallel" condition exists between the bowshock normal and the interplanetary magnetic field. Once energized in such a configuration the particles then move along their trajectories guided by the magnetic field and arrive in the dayside cusp.

Source 2 has the particles being accelerated in situ in the cusp/cleft region by an unknown mechanism in association with deep diamagnetic cavities that have been observed to be coexistent with enhanced fluxes of the energetic particles and the penetration of shocked solar wind inside the magnetopause. Once the particles are energized they are subject to gradient and curvature effects in the geomagnetic field and will drift away from the cusp location.

Source 3 has the particles being energized by processes in the geomagnetic tail associated with substorms. The particles then drift to the dayside where over a narrow range of nightside radial distances they will move into the cusp when drifting to the dayside. This happens because the minimum along a magnetic field line near the dayside magnetopause is no longer at the equator but bifurcates and moves into each of the high altitude cusps. The azimuthal drift motion of these particles will follow the minimum of the magnetic field into either cusp.

Two of these sources (1 and 3) are consistent with the present ideas within the accepted paradigm for the way in which the magnetosphere functions. Source 2 will require a major change in these ideas. All three of these mechanisms may be active to some degree and may generate some energetic particles that show up in the cusp. Rather than argue 
against the role of sources 1 and 3, which, due to geometry alone and the time variable nature of their acceleration process, will have difficulty in producing energetic particles on a continuous basis, this paper will concentrate on source 2, and will present evidence that shocked solar wind ions enter the high altitude, high latitude cusp/cleft from the magnetosheath and are energized very rapidly to $100 \mathrm{~s}$ and $1000 \mathrm{~s}$ of $\mathrm{keV}$. These shocked solar wind ions apparently produce individual diamagnetic cavities in which the magnetic field is observed to decrease from $\sim 100 \mathrm{nT}$ to values approaching zero with a great deal of magnetic field turbulence associated with the cavity (Chen et al., 1997, 1998; Fritz, et al., 1999). The power contained in the fluctuations is correlated to the intensity of the $\mathrm{MeV}$ ions observed (Chen and Fritz, 1998). The mechanism associated with this process seems to be continually active, but individual cavities are impulsive in nature with an event having an apparent lifetime of a few tens of minutes. These facts appear to be well-established by the charge-state, composition, and energy spectral measurements that have been made with Polar, in comparison to simultaneous Geotail measurements in the region upstream and downstream of the bowshock (Chen and Fritz, 1998; Fritz and Chen, 1999a, b).

The proposed cusp mechanism is capable of producing an energetic particle layer that straddles the magnetopause along the flanks of the magnetosphere and of filling the pseudo-trapping region on the dayside of the magnetosphere with energetic particles where they are often observed to exhibit striking energy dispersion signatures (Karra and Fritz, 1999; Fritz et al., 2000). Many authors have reported observations of these energetic particles in the magnetosheath near the magnetopause (Meng and Anderson, 1970; Sarris et al., 1976; Baker and Stone, 1978; Williams et al., 1985). These particles can enter the magnetosphere as a result of gradients in the magnetic field and the resultant drifts they cause; ions entering along the dawn flank drifting to the west and electrons entering along the dusk flank drifting east will carry a current. They provide a rapid coupling of the variations in the subsolar region to the substorm region of the tail magnetosphere. They will also be the source population for the radial diffusion process, which does a very good job of explaining the radial variation of radiation belt fluxes as a function of energy. This result has the potential to be a new paradigm for the way we view the source of particles and flow of information in the magnetosphere.

In this paper we will discuss a particularly fortuitous event on 2-3 May 1998 in the solar wind, which was monitored by the Advanced Composition Explorer (ACE) satellite upstream of the Earth's magnetosphere at the L1 libration point. This event permitted an element of solar wind plasma to be traced into the Earth's magnetosphere, where it will be shown that the Polar satellite observed the same plasma over a series of high latitude passes through the dayside region. A comparison of the phase space density of the same plasma elements at the location of ACE and Polar calculated from these observations provide a definitive indication that the shocked solar wind is energized to $10 \mathrm{~s}, 100 \mathrm{~s}$ and even $1000 \mathrm{~s}$ of $\mathrm{keV}$ in the high latitude subsolar magnetosphere region immediately upon entry into the magnetospheric cusps.

\section{Instrumentation}

The ACE spacecraft was launched on 25 August 1997 and was injected into a halo orbit around the L1 libration point in December 1997. The Solar Wind Ion Composition Experiment (SWICS) (Gloeckler et al., 1998) determines uniquely the chemical and ionic-charge composition of the solar wind, the temperatures and mean speeds of all major solar-wind ions, from $\mathrm{H}$ through $\mathrm{Fe}$, at all solar wind speeds above $300 \mathrm{~km} / \mathrm{s}$ (protons) and $170 \mathrm{~km} / \mathrm{s}(\mathrm{Fe}+16)$, and resolves $\mathrm{H}$ and $\mathrm{He}$ isotopes of both solar and interstellar sources. SWICS measures the distribution functions of both the interstellar cloud and dust cloud pickup ions up to energies of $100 \mathrm{keV} / \mathrm{e}$ and makes a complete measurement of the $\mathrm{Fe}$ ion spectrum in $12 \mathrm{~min}$. Another experiment on ACE is the Electron, Proton, and Alpha Monitor (EPAM) particle instrument (Gold et al., 1998). EPAM is composed of five telescope apertures of three different types. Two Low Energy Foil Spectrometers (LEFS) measure the flux and direction of electrons above $30 \mathrm{keV}$, two Low Energy Magnetic Spectrometers (LEMS) measure the flux and direction of ions greater than $50 \mathrm{keV}$, and the Composition Aperture (CA) measures the elemental composition of the ions. The telescopes use the spin of the spacecraft to sweep the full sky. Solid-state detectors are used to measure the energy and composition of the incoming particles.

Polar was launched into a 1.8 by $9 R_{E}$ orbit on 24 February 1996 with an inclination of 86 degrees. On board Polar, the Magnetospheric Ion Composition Spectrometer (MICS), a part of the Charge and Mass Magnetospheric Ion Composition Experiment (CAMMICE) is a 1-dimensional time-offlight electrostatic analyzer with post acceleration measuring the ions with an energy/charge of 1 to $220 \mathrm{keV} / \mathrm{e}$ with very good angular resolution. The MICS, mounted perpendicular to the spin axis, is able to obtain a 2-dimensional distribution at one energy per charge during each six-second spin period. A complete energy spectrum is obtained in 32 spin periods. The Imaging Proton Spectrometer (IPS), a part of the Comprehensive Energetic Particle and Pitch Angle Distribution (CEPPAD) experiment (Blake et al., 1995) uses an ionimplanted solid-state detector that is discretely segmented into multiple pixels. The detector sits behind a collimation stack at the "focal plane" of a "pin-hole camera", thereby imaging a slice of phase space. Three identical heads, each with three non-overlapping look directions $\left(20^{\circ} \times 12^{\circ}\right)$ provide collectively an instantaneous snapshot of a $180^{\circ} \times 12^{\circ}$ wedge of phase space. As a consequence of spacecraft rotation, the IPS maps out a full 4pi steradian image each spin period $(\sim 6 \mathrm{~s})$. Flux measurements over a nominal energy range from $20 \mathrm{keV}$ to a few $\mathrm{MeV}$ are obtained as a function of pitch-angle and energy each 1/32nd of a spin. The HYDRA experiment (Scudder et al., 1995) is a collection of electrostatic analyzers designed for high resolution observations of 


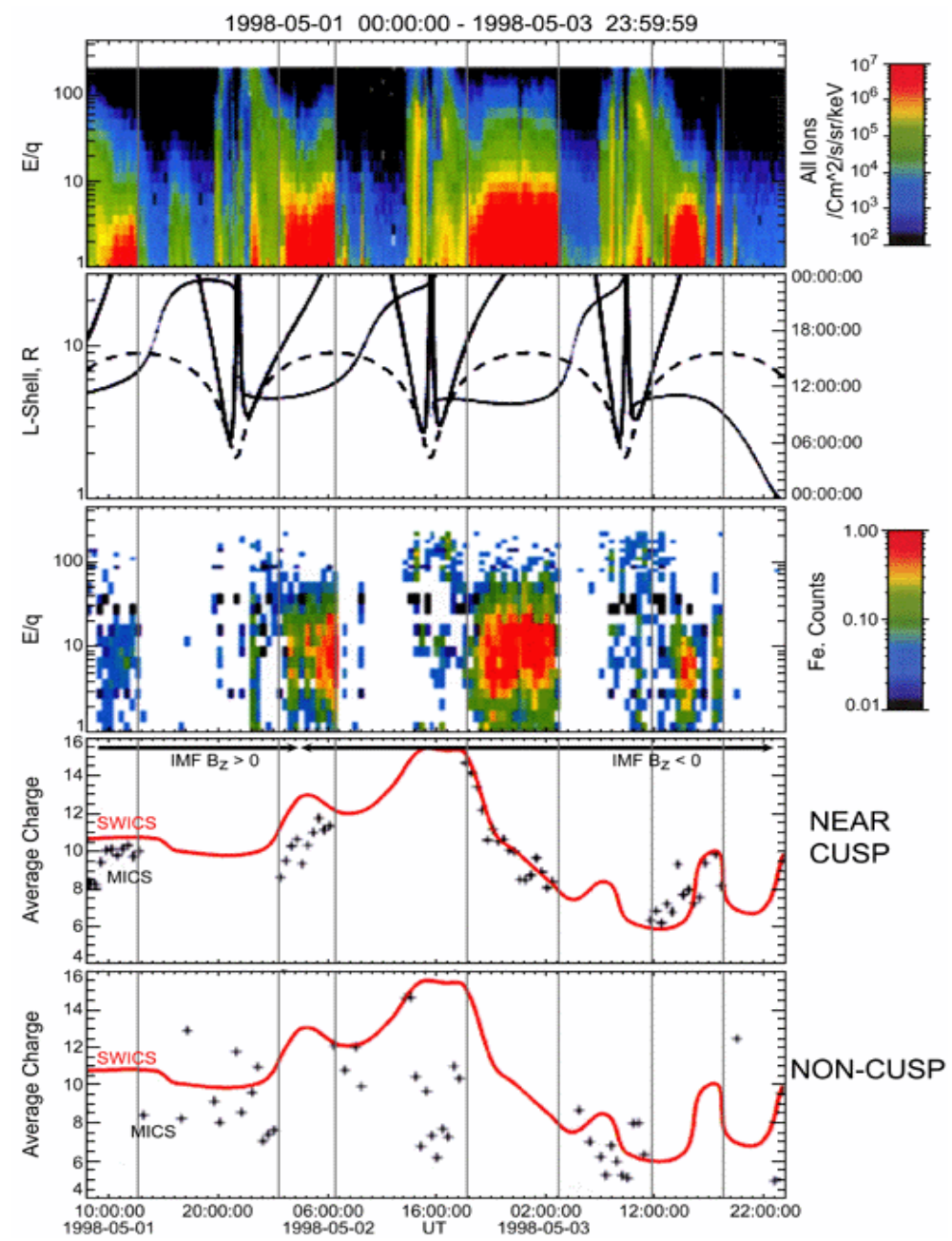

Fig. 1. Polar CAMMICE plasma data for 1-3 May 1998. (a) Total ion intensity, (b) orbital parameters for the Polar satellite showing the radial distance (broken line) and L-shell (heavy solid line) with the scale on the left axis and Magnetic Local Time, MLT (lighter solid line) with scale on the right side axis, (c) the intensity of the Fe ions, (d) the average charge state of the Fe ions measured by the ACE SWICS instrument (red line) and measured by Polar in the region of the cusp/cleft (+), (e) same as above except the Polar measurements are in regions other than the cusp/cleft. This figure has been modified from Fig. 1 of Perry et al. (2000).

electron and ion velocity distributions between $\sim 2 \mathrm{keV} / \mathrm{e}$ and $35 \mathrm{keV} / \mathrm{e}$ in three dimensions with a routine time resolution of $0.5 \mathrm{~s}$. Using other operational modes of the Hydra experiment it is possible to extend the lower limit of the measurement to $10 \mathrm{eV} / \mathrm{e}$.

\section{Observations and discussion}

During the period of 2-3 May 1998, the SWICS instrument on the ACE satellite at the L1 libration point observed a variation in the charge state of the solar wind $\mathrm{Fe}$ ions that ranged from an average value of +6 to +15 (Gloeckler et al., 1999). At the same time the Polar satellite observed a similar series of changes in the charge state of $\mathrm{Fe}$ ions measured inside the magnetosphere in the vicinity of the dayside high altitude cusp. The comparison of measurements from the two satellites has been reported by Perry et al. (2000), and here, Fig. 1 is a slightly modified version of Fig. 1 from their paper. Perry et al. discussed the measurement techniques on the two satellites, the implication of the variation of Fe charge state in the solar wind, and provide a comparison of the average $\mathrm{Fe}$ charge states. In Fig. 1 the measurement at ACE are one-hour averages and are represented by the red curve. The measurements at the position of Polar are given as the plotted "+". The time scale for the ACE measurements was corrected for the propagation time from the L1 point to the subsolar magnetopause based on the measured solar wind velocity. Note that over the two and a half day period presented in Fig. 1 the average charge state of $\mathrm{Fe}$ ions measured by ACE varied initially from a value of +10 , rising to +15 and then transitioning down to +6 . This corresponded to changes in the coronal source temperature of $4 \times 10^{5}$ to $2 \times 10^{6} \mathrm{~K}$.

Perry et al. (2000) have reported these measurements and their relationship to the timing of the arrival of a CME and some companion geomagnetic activity that followed. They concluded that during the period of southward interplanetary magnetic field indicated in Fig. 1 a very good correlation between the measurements at ACE and at Polar was found, indicating that both spacecraft were sampling the same CME plasma population. The time scales of these observations were consistent with direct and rapid entry of solar wind plasma into the magnetosphere, concluding that this entry was via a subsolar magnetic field line merging site. In Fig. 1 there are four periods when Polar was on the dayside at high 




Fig. 2. The phase space density of the total ion population determined by the SWICS and EPAM instruments at ACE and by the HYDRA, MICS, and IPS instruments on Polar, as a function of energy/charge. The two populations correspond to a plasma with an average Fe charge state of +15 recorded just before 19:00 UT on 2 May at ACE and just after 19:00 UT at Polar.

latitude and high altitudes, labeled as "near cusp". The comparison of the Fe charge state during these periods is given in the fourth panel, and a fifth panel is included showing the same comparison during the "non-cusp" periods. Perry et al. (2000) presented the details of the Polar cusp/cleft periods in their Table 1. That table is reproduced here as Table 1 with an added column for the difference of the Start and End times. The feature to note in the table is the extended period of time in which Polar was located in the cusp/cleft or rather in a shocked solar wind plasma population that had recently and directly entered the magnetosphere. These observations are not consistent with the concept of a narrow funnel-shape cusp geometry. Fritz et al. (2003) have shown that contact by the Polar satellite with a very wide extended region of solar wind plasma entry is a common occurrence along the orbit of Polar, when the orbital plane is within a few hours of local noon.

From the response of the instruments on the ACE spacecraft and on the Polar satellite it is possible to construct an energy spectrum from about $1 \mathrm{keV} / \mathrm{e}$ to beyond a few $\mathrm{MeV}$ for the dominant proton particle species. On Polar this range can be extended below $0.1 \mathrm{keV} / \mathrm{e}$ by the Hydra instrument. Selecting period \#3 in Table 1 and Fig. 1 the average value
Table 1. Details of the Polar cusp/cleft periods

\begin{tabular}{ccllccc}
\hline Period & Date & $\begin{array}{l}\text { Start } \\
\text { UT }\end{array}$ & $\begin{array}{l}\text { End } \\
\text { UT }\end{array}$ & $\begin{array}{c}\text { Difference } \\
\text { (hours) }\end{array}$ & $\begin{array}{c}\text { L1 } \\
\text { (degs) }\end{array}$ & $\begin{array}{c}\text { L2 } \\
\text { (degs) }\end{array}$ \\
\hline 1 & 1-May & $10: 30$ & $13: 00$ & 2.5 & 80.3 & 85.9 \\
2 & 2-May & $2: 30$ & $7: 00$ & 4.5 & 73.3 & 82.0 \\
3 & 2-May & $20: 00$ & $3: 00$ & 7.0 & 75.8 & 85.4 \\
4 & 3-May & 13:30 & $16: 00$ & 2.5 & 76.4 & 83.3 \\
\hline
\end{tabular}

of the iron charge state is observed to vary from +15 down to +8 over the duration of the seven-hour period where Polar continuously observed the shocked solar wind plasma. The tracking of the Fe charge states in the two data sets indicates that both are observing the same solar wind plasma. In other words, an element of solar wind plasma is "tagged" as it passes ACE and is then observed by Polar inside the magnetosphere with delays that are only associated with the solar wind propagation from ACE to the subsolar magnetopause. From the measured values of the particle fluxes, $j$ (particles $/ \mathrm{cm}^{2} \mathrm{~s}$ sterradian $\mathrm{keV}$ ) as a function of energy, the phase space density, $f\left(\mathrm{~s}^{3} / \mathrm{m}^{6}\right)=5.45 \times 10^{-31} j /$ Energy $(\mathrm{keV})$ can be calculated at the location of the two spacecraft. In the absence of sources and losses, Liouville's theorem states that the density of particles in phase space, $f$, is constant along the particle trajectory.

Figure 2 compares the phase space densities as a function of energy/charge measured at the two spacecraft for the same "tagged" element of plasma. The two populations correspond to plasma with an average Fe charge state of +15 averaged for the 30-min period just before 19:00 UT on 2 May at ACE and averaged for the 30-min period following 19:00 UT at Polar. The comparison in Fig. 2 also indicates that the plasma has not only been thermalized, but it has been energized significantly along its trajectory from ACE to Polar over a range of energy from a few $\mathrm{keV}$ to about one $\mathrm{MeV}$. Note the agreement and consistency of the responses of the independently calibrated sensors on the two spacecraft. The particle energization at constant phase space density is between a factor of five and ten. More significantly, however, is the fact that an instrument with a constant energy threshold would observe about a three order of magnitude increase in the particle flux it would measure as a result of this energization. In the comparison of Fig. 2 the maximum in phase space density is the peak in the solar wind, and the solar wind fluxes are fully capable of being the source of the population measured at Polar.

A comparison of the phase space densities at the two spacecraft using an element of plasma tagged with an average Fe charge state of +10 is presented in Fig. 3. The ACE data are averaged over the 30-min period prior to 23:00 UT, and the Polar data are averaged over the 30-min period after 23:00 UT. There has been little change in the solar wind phase space density measured at ACE, with the peak at about $3 \times 10^{7} \mathrm{~s}^{3} \mathrm{~km}^{-6}$. The phase space densities at the position of Polar show the same general characteristics but are different 


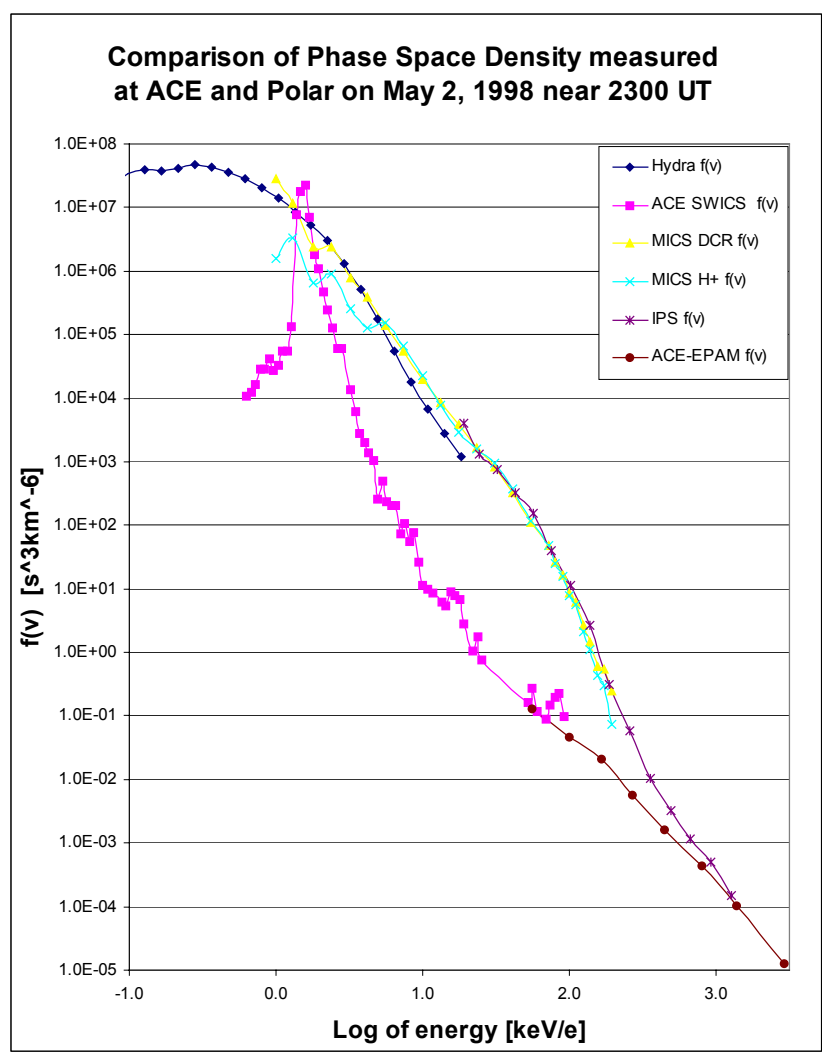

Fig. 3. Same format as Fig. 2 but now the two populations correspond to a plasma with an average Fe charge state of +10 recorded just before 23:00 UT on 2 May at ACE and just after 23:00 UT at Polar.

in a fundamental way. The thermalization and significant energization of the plasma is again evident but now the peak in the phase space density at ACE is less than that at Polar. The solar wind plasma is not fully capable of supplying all of the particles measured by Polar. There must be an additional source of plasma at Polar. The most likely source of this additional plasma population is the Earth's ionosphere, and the most likely such ion is $\mathrm{O}^{+}$that can flow out of the ionosphere via the cleft ion fountain (Lockwood et al., 1985; Moore et al., 1985).

The CAMMICE MICS sensor has the capability to examine the charge-state composition of the energized plasma to determine if there might be fluxes of ions of ionospheric origin. In Fig. 4 the response of the MICS sensor to all major ion species is presented. The top panel displays oxygen ions with high charge-state oxygen, indicative of ions that originate in the solar wind. In the second panel the fluxes of oxygen ions with charge states associated with an ionospheric source are displayed. The threshold energy of $\sim 50 \mathrm{keV}$ for the detection of these $\mathrm{O}^{+}, \mathrm{O}^{++}$ions is much higher than that of $\mathrm{O}^{+6}$ shown in the first panel. The reason for this is that the MICS energy measurement is done by a solid-state detector with a contact dead layer that must be penetrated by the respective ion. In the case of $\mathrm{O}^{+6}$ the post acceleration of $20 \mathrm{KV}$ provides $120 \mathrm{keV}$ of addition energy to the ion permitting the full range of these ion energies to be measured, if desired. In the case of $\mathrm{O}^{+}$they receive only an additional $20 \mathrm{keV}$ of energy, which is not sufficient to penetrate the detector contact, resulting in an energy threshold of $\sim 50 \mathrm{keV}$. The third and fourth panels display the fluxes of helium ions, with the third panel being alpha particles and the fourth panel being singly charged helium. The fifth panel shows the total $\mathrm{H}$ or hydrogen intensity. We see the seven-hour period from 19:00 h on 2 May to $03: 00 \mathrm{~h}$ on 3 May easily identified with intense fluxes of high charge-state oxygen, alpha particles and hydrogen. The magnetic field for this interval is displayed in the lower-right inset of Fig. 4. During this interval, Polar moves from a region of northward pointing magnetic field, through a very turbulent, depressed, and highly variable region but variable around a constant level, until the satellite leaves the depressed field region where its encounters a field directed earthward. The $Z$ component was negative throughout the highly turbulent region associated with this shocked solar wind plasma.

It is possible with the CAMMICE MICS to evaluate the phase space densities as a function of their parallel and perpendicular velocities. For the interval covered by the data in Figs. 2 and 3 and in between, we display in Fig. 5 the phase space distribution at all energies in the top portion of the panel, and we zoom in on the distribution at lower energies in the bottom panel. Note that the distribution at 19:15 h is basically an isotropic distribution with a small preference for 90-degree pitch angles as the distribution evolves in time. The preference for a trapped distribution continues, but toward the end of the sequence, the distribution still takes on a broad, hemispherical distribution, with the peak at v-perpendicular but indicating that the direction away from the Earth was unable to maintain a trapping geometry. The lack or decrease of particle fluxes in the direction parallel to the magnetic field is consistent with the conclusions that the energized fluxes are coming from the direction of the Earth.

Returning to the observations in Fig. 3 in that an additional source of ions is required to supply the fluxes or phase space density seen at Polar during this interval, we see in Fig. 4 that ions most likely of ionospheric origin are present. These are $\mathrm{He}^{+}$and $\mathrm{O}^{+}$. It is possible to examine the ion composition Polar data in greater detail. In Fig. 6 we display the ion composition data for the period of Fig. 2 using only those data that produce a valid time-of-flight (TOF) without a companion valid energy response. This display will permit the MICS instrument to examine the lowest energy oxygen ions that it is capable of detecting. We show in the top panel those ion responses that are restricted to "zero energy". When these responses are analyzed, looking now at the TOF versus $E / q$ panel, we see that there are basically three main ion groups: $\mathrm{H}^{+}, \mathrm{He}^{++}$, and $\mathrm{O}^{+}$. There is also some $\mathrm{He}^{+}$but the $\mathrm{O}^{+}$ clearly stands out as a line on top of the background, indicating a significant $\mathrm{O}^{+}$population. Note also the energy of the $\mathrm{O}^{+}$ions depicted by the $E / q$ axis which indicates that these ions out of the ionosphere had energies from 1 to $100 \mathrm{keV}$. The energies indicate that these $\mathrm{O}^{+}$ions have undergone significant energization from their parent population 


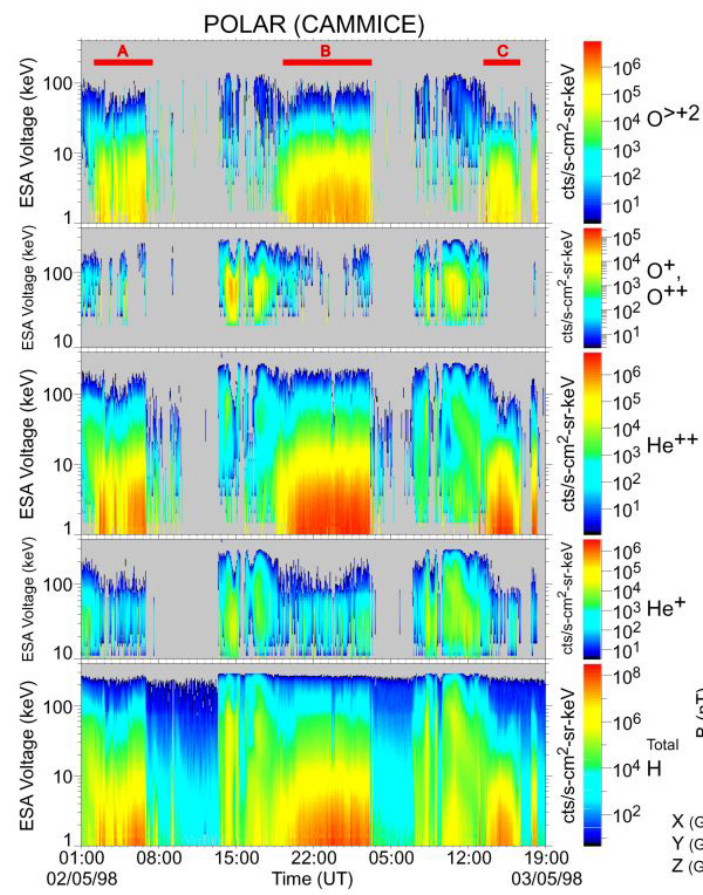

\section{2-3 May 1998}
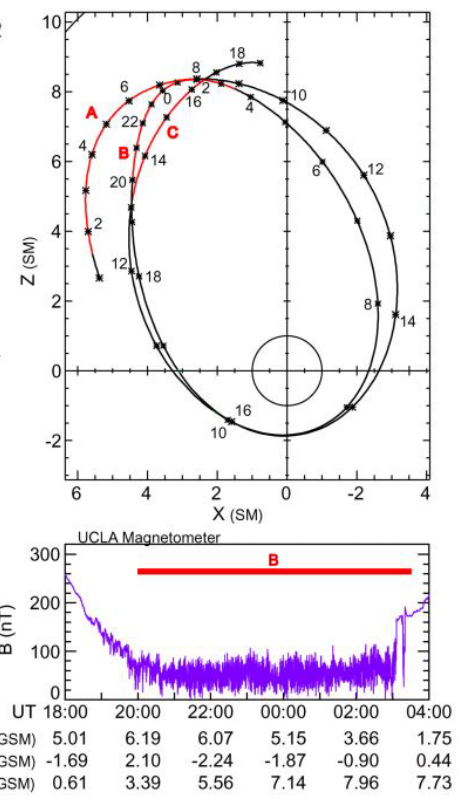

Fig. 4. The intensity of the ion species, $\mathrm{H}^{+}, \mathrm{He}^{++}, \mathrm{He}^{+}, \mathrm{O}^{+}$, and high charge state $\mathrm{O}$, as a function of particle energy for the period of 2 and 3 May 1998. Note the three periods in which an extended signature of high chargestate $1-10 \mathrm{keV}$ ions was seen along the trajectory of the Polar satellite. The orbit projected onto the SM $X Z$-plane is also shown with an indication of where these extended signatures are seen. For the interval of Period 3 in Table 1 an inset showing the variation of the total magnetic field is shown (courtesy of C. T. Russell), demonstrating the depressed magnetic field associated with the extended signature of high chargestate ions.
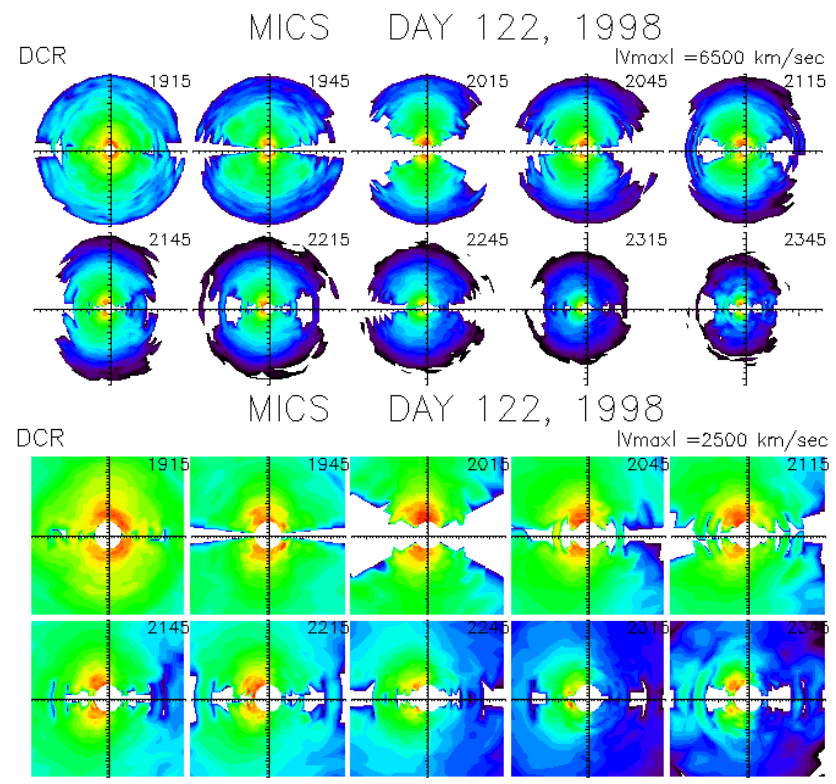

Fig. 5. The phase space distribution at all energies as a function of the parallel and perpendicular particle velocities in the top portion of the panel and the same distribution at lower energies in the bottom panel determined using the double coincidence response [DCR] of the Polar MICS senor.

in the ionosphere. It seems likely that the energization process for the solar wind ions and these ionospheric ions is located at the same place. We believe the energization of both parent populations to energies equivalent to those responsible for the ring current occurs in the diamagnetic cavities associated with the depressed field regions observed to be coexistent with the shocked solar wind plasma intrusion inside the magnetosphere (Chen and Fritz, 2001). In Fig. 7 the detailed energy spectrum on each of the ion species measured by Polar is presented. The curve labeled $\mathrm{O}_{\mathrm{o}}$ is for oxygen ions with charge states associated with a source in the ionosphere determined with the "zero energy" technique described above. As determined previously from Fig. 6 there are large fluxes of very energetic oxygen ions of ionospheric origin coexisting with the energized ions of solar wind origin during the time period from 19:00-19:30 UT. The fluxes of $\mathrm{O}_{0}$ above $6 \mathrm{keV} / \mathrm{e}$ are equivalent to those of solar wind $\mathrm{He}^{++}$and are the dominant oxygen ion during this period. The detailed energy spectra for ions measured during the period from 23:0023:30 UT is displayed in Fig. 8. Again, there were appreciable fluxes of oxygen ions of ionospheric origin present. They were nearly equivalent but were not the dominant oxygen ion species in this case for energies above $2 \mathrm{keV} / \mathrm{q}$; at energies of $1 \mathrm{keV} / \mathrm{q}$ and below they probably dominate the helium and higher charge-state oxygen ions. This is most likely why the phase space density at Polar in the region below $1 \mathrm{keV} / \mathrm{q}$ exceeds the phase space density of the solar wind source ions.

In Fig. 9 the phase space densities measured at the ACE spacecraft and at Polar at 02:00 UT on 3 May are compared. The ACE measurements were averaged for the 30 -min period prior to 02:00 UT and the Polar measurements were already for the 30-min interval following 02:00 UT. Again, we observe that the comparison of phase space densities indicates that thermalization and significant energization had occurred in the fluxes measured at Polar. Again, the phase space density measured by the ACE spacecraft was unable to supply all of the particles needed to generate the phase space densities measured at Polar at this time. The average charge state of the $\mathrm{Fe}$ ions is +8 during this interval. When the detailed ion energy spectra are examined (Fig. 10) we again 


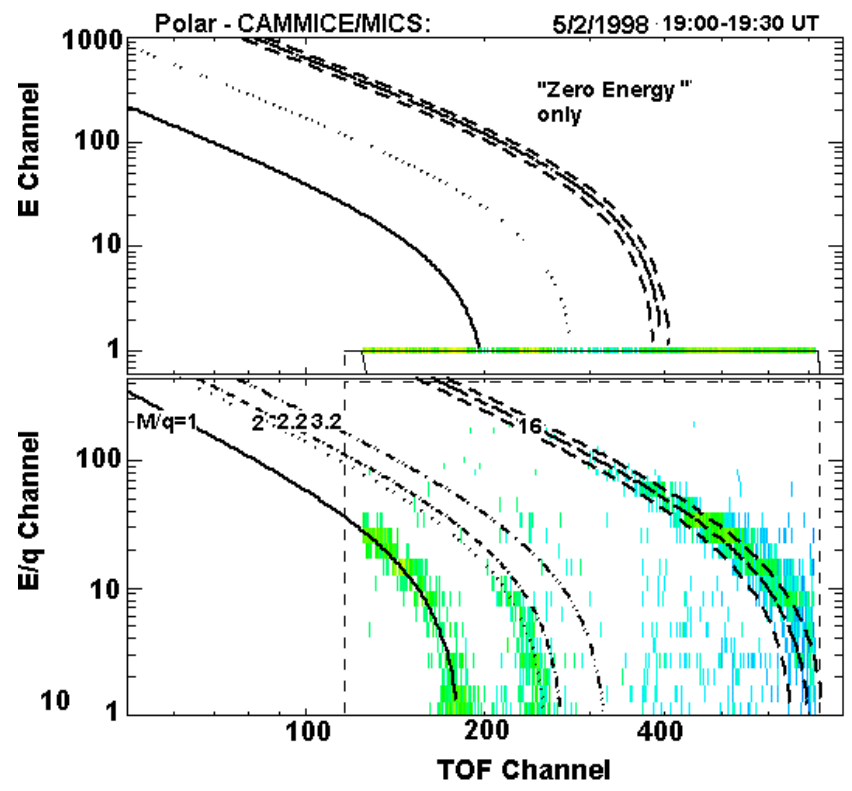

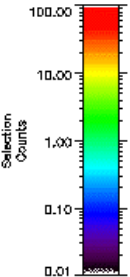

(100.00

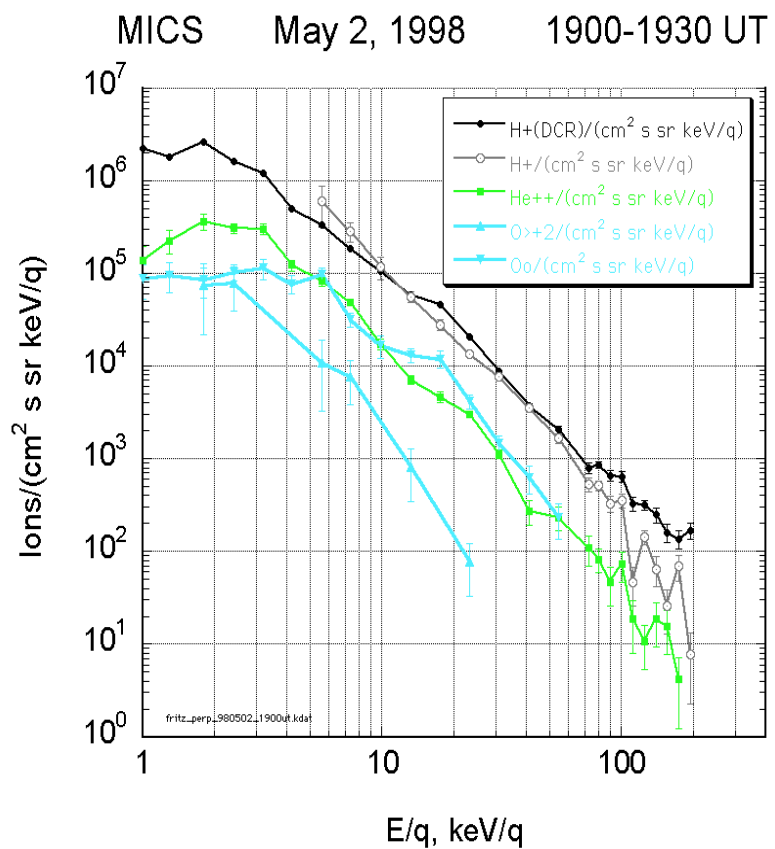

Fig. 7. The energy spectrum of flux versus energy/charge of the four major ion species determined for the interval from 19:00 UT to 19:30 UT on 2 May 1998.

see a significant presence of ions of ionospheric, as well as solar wind origin. The tendency noted above for the ionospheric ions to become the dominant minor ion species below $1 \mathrm{keV} / \mathrm{q}$ is again consistent with the fact that the phase space density at Polar below $1 \mathrm{keV} / \mathrm{q}$ dominated those measured by ACE upstream in the solar wind.

As can be seen in the Fig. 1, around 03:00 UT, Polar left local times associated with the dayside and proceeded into
Fig. 6. Display of the ion composition direct event data for the period of Fig. 2 using only those data that produce a valid time-of-flight (TOF) without a companion valid energy response.

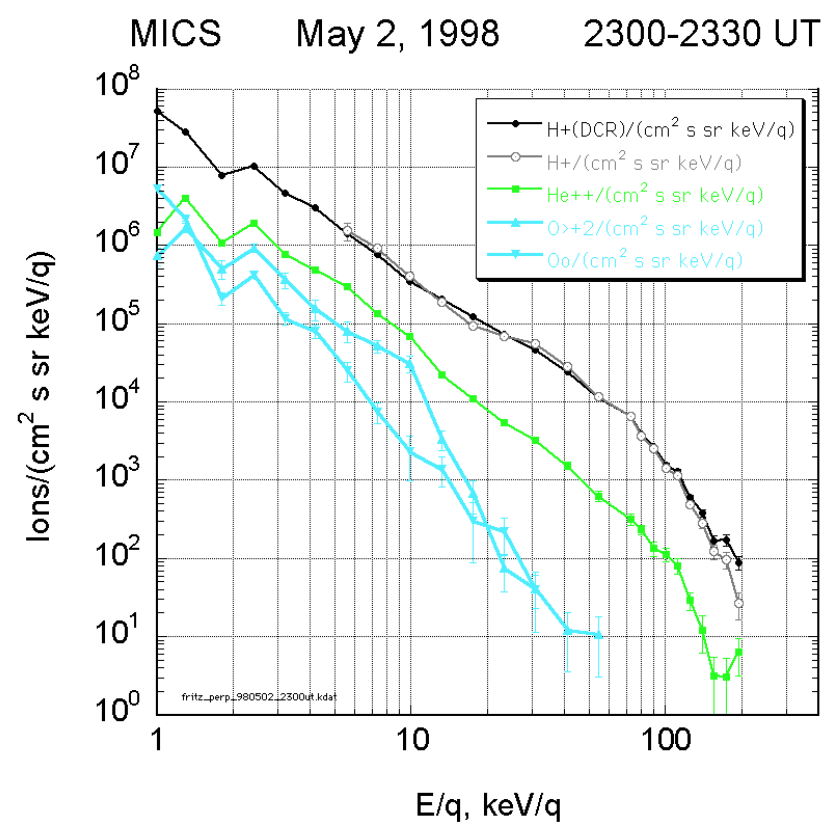

Fig. 8. The energy spectrum of flux versus energy/charge of the four major ion species determined for the interval from 23:00 UT to 23:30 UT on 2 May 1998.

the nightside magnetosphere, leaving at the same time the region of shocked solar wind plasma. The Polar satellite moved down through the radiation belts, over the southern polar regions, back out through the radiation belts, and again entered the high latitude dayside region in the vicinity of the magnetospheric cusp. Slightly after 13:00 UT it again encountered an extended region of shocked solar wind plasma, noted as Period 4 in Table 1. When the Fe charge state was deter- 


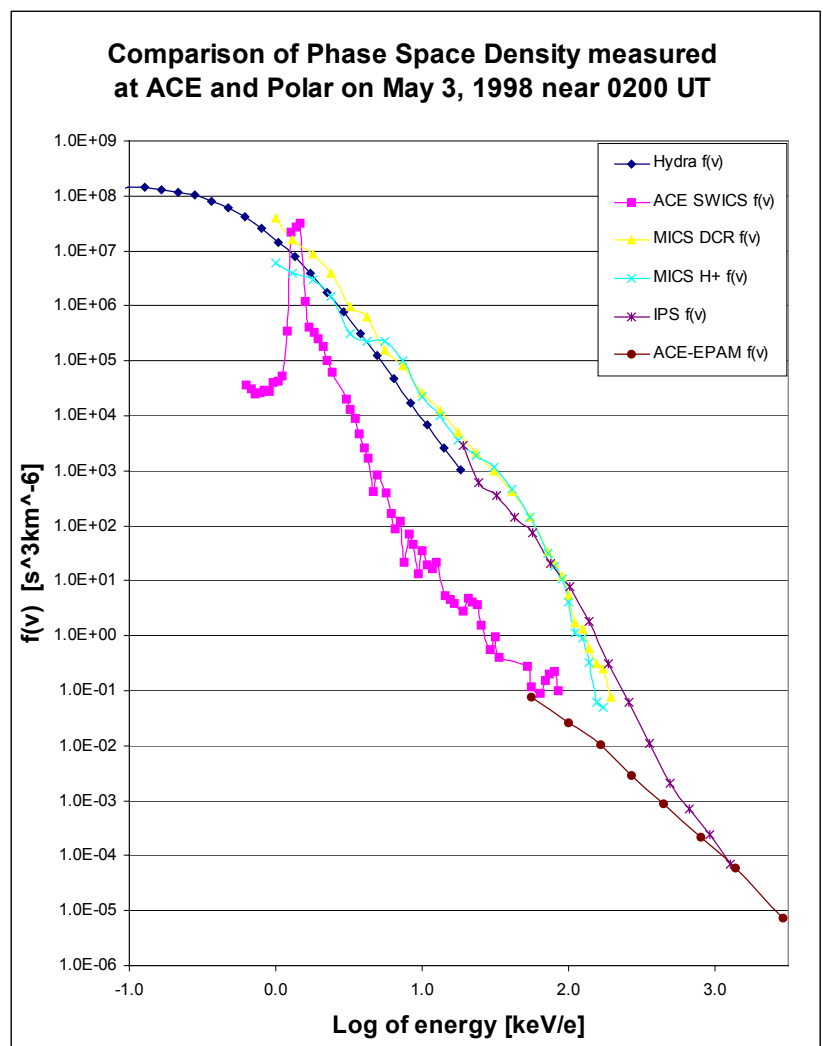

Fig. 9. Same format as Fig. 2 but now the two populations correspond to a plasma with an average $\mathrm{Fe}$ charge state of +8 recorded just before 02:00 UT on 2 May at ACE and just after 02:00 UT at Polar.

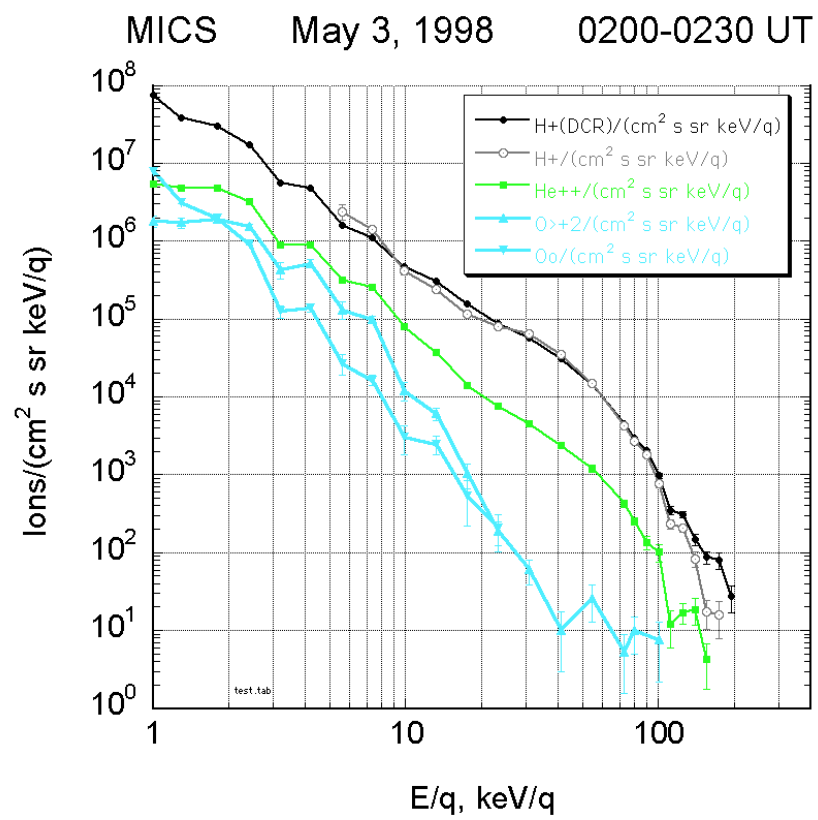

Fig. 10. The energy spectrum of flux versus energy/charge of the four major ion species determined for the interval from 02:00 UT to 02:30 UT on 3 May 1998.

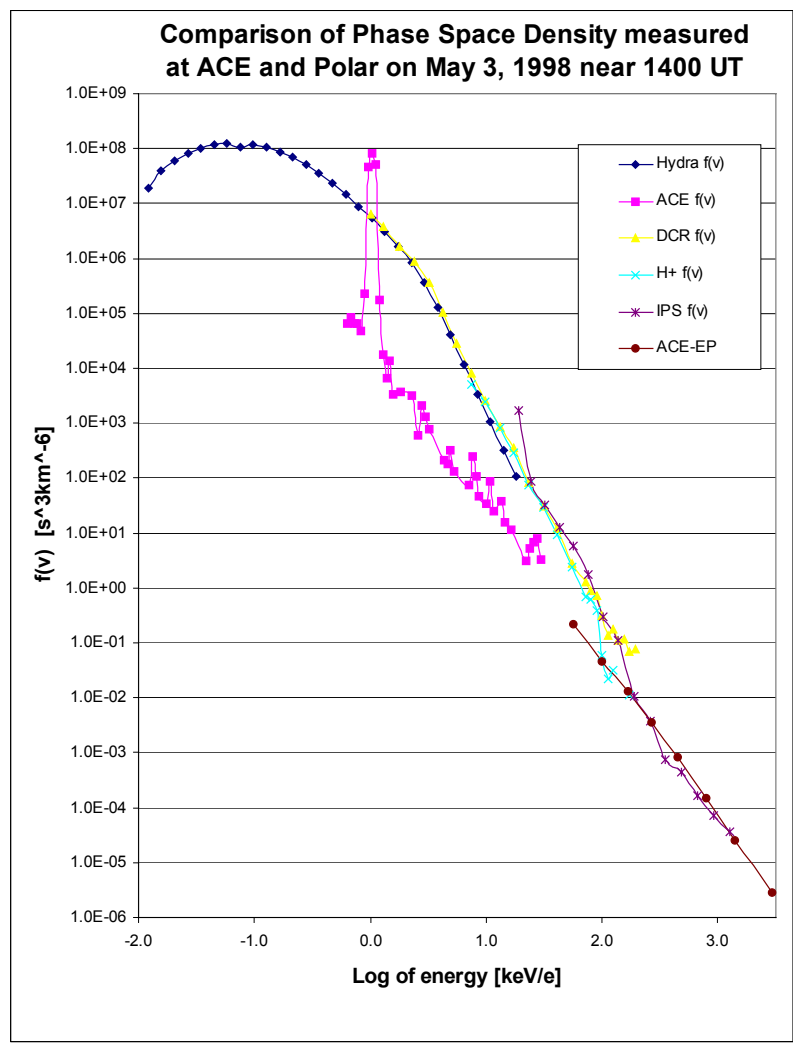

Fig. 11. Same format as Fig. 2 but now the two populations correspond to a plasma with an average Fe charge state of +6 recorded just before 14:00 UT on 3 May at ACE and just after 14:00 UT at Polar.

mined it again followed in time that measured upstream by the ACE spacecraft. A comparison was made for the same plasma element, this time with an average $\mathrm{Fe}$ charge state of six to seven at the two spacecraft near 14:00 UT. This comparison of phase space densities measured at two spacecraft is presented in Fig. 11 with the same thermalization and significant energization occurring at Polar, but again the phase space densities measured by ACE were inadequate to provide the necessary particles measured by the Polar spacecraft. This region of shocked solar wind plasma with the ion composition dominated by ions $\mathrm{H}^{+}, \mathrm{He}^{++}$and high chargestate oxygen was apparent in the interval from 14:00 UT to beyond 18:00 UT in Fig. 4. From Fig. 1 we see that Polar stayed in the region of shocked solar wind plasma until the spacecraft moved from those local times associated with the dayside toward local times associated with the nightside magnetosphere. Figure 12 displays the detailed energy spectra at Polar for the major and minor ion species. Although $\mathrm{He}^{++}$was the dominant minor ion species at all energies, there were significant fluxes of low charge-state ionospheric ions at all energies that displayed a similar spectral shape when compared to the species from the solar wind.

During the intervals analyzed in Figs. 2, 3, 9, and 11, we have observed a consistent pattern in which an element of plasma was followed from the solar wind at the L1 libration 


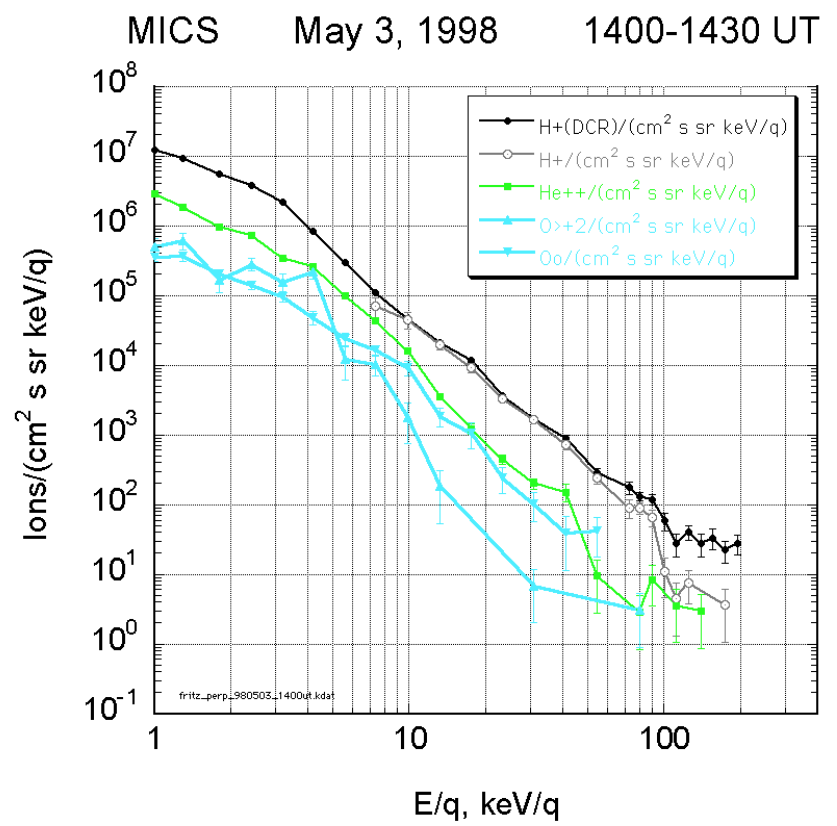

Fig. 12. The energy spectrum of flux versus energy/charge of the four major ion species determined for the interval from 14:00 UT to 14:30 UT on 3 May 1998.

point to the Polar spacecraft inside the magnetosphere. In each of these cases the interplanetary magnetic field (IMF) had a southward component. This geometry favors subsolar reconnection and easy entry of such plasma into the magnetosphere. There was a period earlier on 2 May when the interplanetary magnetic field had a northward geometry that would not favor subsolar reconnection and easy plasma entry. If Fig. 4 is examined, Polar was in a similar cusp-like environment from 02:00 UT until almost 07:00 UT, observing a shocked solar wind plasma (Period 2 in Table 1), and we see in Fig. 1 that the close association in time of the $\mathrm{Fe}$ charge-state variation at ACE and at Polar is no longer apparent. There appears to be a similar slope to the variation of the charge state with time, but with a delay between those measurements made at Polar compared with those at the ACE spacecraft. It is instructive to examine the phase space densities at the two locations during this period. The interval around 06:00 UT was chosen for such a comparison. This is shown in Fig. 13 and again, we see that the plasma was energized upon entry into the magnetosphere, but the entry was delayed by a significant time interval of a few hours. In Fig. 1 the sign of the IMF $B_{z}$ component is displayed at the top of the fourth panel in the figure. The role of reconnection for these observations appears to be that of a gatekeeper. When reconnection is favored the plasma enters the magnetosphere and is immediately energized. When subsolar reconnection is not favored the plasma still enters into the magnetosphere and is energized but with a time delay.

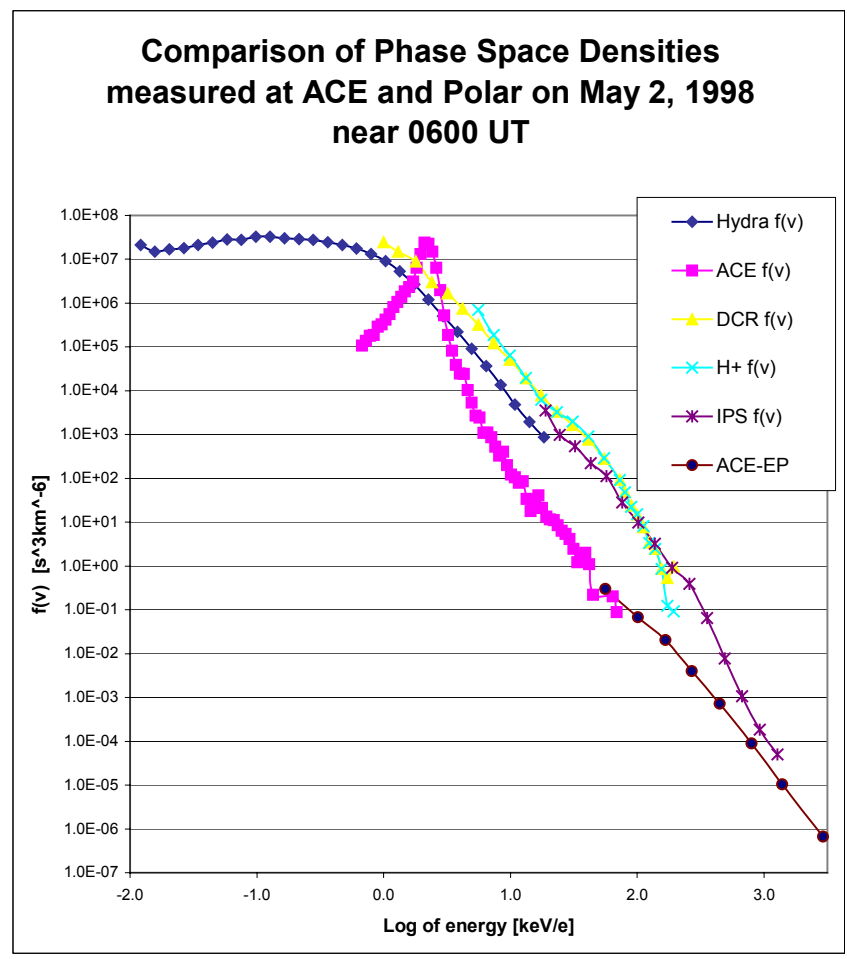

Fig. 13. Same format as Fig. 2 but now the two populations correspond to a plasma recorded just before 06:00 UT on 2 May at ACE and just after 06:00 UT at Polar. Even though the charge state comparison is not necessarily unique, as in the previous cases, there is still the same general behavior of the two measured plasma populations.

\section{Summary}

We have observed a consistent pattern in which an element of plasma has been followed from the solar wind at the L1 libration point to the Polar spacecraft inside the magnetosphere. We have seen that this plasma becomes significantly energized immediately upon entry into the magnetosphere and in most cases the resultant plasma population is a mixture of those solar wind ions with ions of ionospheric origin. These ions are energized over a broad energy range from one $\mathrm{keV} / \mathrm{q}$ up to energies in excess of one MeV. These energies and particle intensities are characteristic of the observed ring current spectrum measured on the nightside of the equatorial magnetosphere near six Earth radii (Fritz et al., 2000). Once energized these ions will be capable of executing gradient and curvature drift in the geomagnetic field and will execute various drift orbits, known as a "Shabansky orbit" (Fritz, 2000) or they will leave the magnetosphere along open field lines and will form a layer of energetic particles on the magnetopause. The nature of the cusp-associated acceleration process of these ions will require further study.

Acknowledgements. We want to acknowledge the contributions of the various instrument teams for CAMMICE, CEPPAD, and Hydra on Polar and SWICS and EPAM on ACE whose hard work produced the well-calibrated sensors that are compared in this paper. 
The effort of J. Fennell, M. Grande, and C. Perry in the determination of the ion composition used in the MICS spectral analysis and Reiner Friedel in supplying the Hydra phase space densities is gratefully acknowledged. The Polar effort has been supported at Boston University under a series of NASA grants: NAG5-2578, NAG5-7677, and NAG5-11397.

Topical Editor T. Pulkkinen thanks I. Daglis for his help in evaluating this paper.

\section{References}

Baker, D. N., and Stone, E. C.: The magnetopause energetic electron layer 1.Observations along the distant magnetotail, J. Geophys. Res. 83, A9, 4327, 1978.

Blake, J. B., Fennell, J. F., Friesen, L. M., Johnson, B. M., Kolasinski, W. A., Mabry, D. J., Osborn, J. V., Penzin, S. H., Schnauss, E. R., Spence, H. E., Baker, D. N., Belian, R. D., Fritz, T. A., Ford, W., Laubscher, B., Stiglich, R., Baraze, R. A., Hilsenrath, M. F., Imhof, W. L., Kilner, J. R., Mobilia, J., Voss, D. H., Korth, A., Guell, M., Fisher, K., Grande, M., and Hall, D.: CEPPAD, Comprehensive Energetic Particle Pitch Angle Distribution experiment, Space Sci. Rev., 71, 531-562, 1995.

Chen, J., Fritz, T. A., Sheldon, R. B., Spence, H. E., Spjeldvik, W. N., Fennell, J. F., and Livi, S.: A new, temporarily confined population in the polar cap during the August 27, 1996 geomagnetic field distortion period, Geophys. Res. Lett., 24 (12), 1447-1450, 1997.

Chen, J. and Fritz, T. A.: Correlation of cusp MeV helium with turbulent ULF power spectra and its implications, Geophys. Res. Lett., 25, 4113, 1998.

Chen, J., Fritz, T. A., Sheldon, R. B., Spence, H. E. Spjeldvik, W. N., Fennell, J. F., Livi, S., Russell, C. T., Pickett, J. S., and Gurnett, D. A.: Cusp energetic particle events: Implications for a major acceleration region of the magnetosphere, J. Geophys. Res., 103 (No. A1), 69-78, 1998.

Chen, J. and Fritz, T. A.: Energetic oxygen ions of ionospheric origin observed in the cusp, Geophys. Res. Lett., 28, 1459-1462, 2001.

Fritz, T. A., Chen, J., Sheldon, R. B., Spence, H. E., Fennell, J. F., Livi, S., Russell, C. T., and Pickett, J. S.: Cusp energetic particle events measured by POLAR spacecraft, Phys. Chem. Earth (C), 24, 135-140, 1999.

Fritz, T. A. and Chen, J.: The Cusp as a sources of Magnetospheric Particles, in: Proceeding of the Workshop on "Space Radiation Environment Modeling: New Phenomena and Approaches" which was held in Moscow Russia on October 7-9, 1997 published in Radiation Measurement, 30, No. 5, 599-608, 1999a.

Fritz, T. A. and Chen, J.: Reply to "Comment on:'Correlation of cusp MeV helium with turbulent ULF power spectra and its implications"' by Trattner, Fuselier, Peterson, and Chang, Geophys. Res. Lett., 26, No. 10, 1363, 1999 b.

Fritz, T.A., Chen, J., and Sheldon, R. B.: The Role of the Cusp as a Source for Magnetospheric Particles: A New Paradigm? Adv.
Space Res., 25, No 7-8, 1445-1457, 2000.

Fritz, T. A.: The Role of the Cusp as a Source for Magnetospheric Particles: A New Paradigm, ESA Special Publication of the Proceedings of the Cluster II Workshop on Multiscale/Multipoint Plasma Measurements held at Imperial College, London 22-24 September 1999, ESA SP-499, February, 2000.

Fritz, T. A., Chen, J., and Siscoe, G. L.: Energetic ions, large diamagnetic cavities, and Chapman-Ferraro cusp, J. Geophys. Res., 108(A1), 1028-1036, 2003.

Gold, R. E., Krimigis, S. M., Hawkins, S. E., Haggerty, D. K., Lohr, D. A., Fiore, E., Armstrong, T. P., Holland, G., and Lanzerotti, L. J.: Electron, Proton and Alpha Monitor on the Advanced Composition Explorer Spacecraft, Space Sci. Rev., 86, 541, 1998.

Gloeckler, G., Cain, J., Ipavich, F. M., Tums, E. O., Bedini, P., Fisk, L. A., Zurbuchen, T. H., Bochsler, P., Fischer, J., WimmerSchweingruber, R. F., Geiss, J., and Kallenbach, R.: Investigation of the Composition of Solar and Interstellar Matter Using Solar Wind and Pickup Ion Measurements with SWICS and SWIMS on the ACE Spacecraft, Space Sci. Rev., 86, 457-539, 1998.

Gloeckler, G., Hefti, S., Zurbuchen, T. H., Schwadron, N. A., Fisk, L. A., Ipavich, F. M., Geiss, J., Bochsler, P., and Wimmer, R.: Unusual Composition of the Solar Wind in the 2-3 May 1998 CME Observed With SWICS on ACE, Geophys. Res. Lett., 26, 157-160, 1999.

Karra, M. and Fritz, T. A.: Energy Dispersion Features in the Vicinity of the Cusp, Geophys. Res. Ltrs., 26, 3553, 1999.

Lockwood, M., Chandler, M. O., Horwitz, J. L., Waite, Jr., J. H., Moore, T. E., and Chappell, C. R.: The cleft ion fountain, J. Geophys. Res., 90, 9736, 1985.

Meng, C.-I. and Anderson, K. A.: A layer of energetic layer (>40 Kev) near the magnetopause, J. Geophys. Res. 75, 1827, 1970.

Moore, T. E., Chappell, C. R., Lockwood, M., and Waite, Jr., J. H.: Superthermal ion signatures of auroral acceleration processes, J. Geophys. Res., 90, 1611, 1985.

Perry, C. H., Grande, M., Zurbuchen, T. H., Hefti, S., Gloeckler, G., Fennell, J. F., Wilken, B., and Fritz, T. A.: Use of Fe charge states as a tracer for solar wind entry to the magnetosphere, Geophys. Res. Ltrs., 27, 2441-2444, 2000.

Sarris, E. T., Krimigis, S. M., and Armstrong, T. P.: Observation of magnetospheric bursts of high-energy protons and electrons at $\sim 35 R_{E}$ with IMP-7, J. Geophys. Res. 81, No. A13, 2341, 1976.

Scudder, J., Hunsacker, F., Miller, G., Lobell, J., Zawistowski, T., Ogilvie, K., Keller, J., Chornay, D., Herrero, F., Fitzenreiter, R., Fairfield, D., Needell, J., Bodet, D., Googins, J., Kletzing, C., Torbert, R., Vandiver, J., Bentley, R., Fillus, W., Mcllwain, C., Whipple, E., and Korth, A.: Hydra-A 3-dimensional electron aand ion hot plasma instrument for the Polar spacecraft of the GGS mission, Space Sci. Rev., 71, 459-495, 1995.

Williams, D. J., Mitchell, D. G., Eastman, T. E., and Frank, L. A.: Energetic particle observations in the low latitude boundary layer, J. Geophys. Res. 90, 5097, 1985. 